\title{
Gaze Perception Develops Atypically in Children with Autism
}

\author{
Simon Webster and Douglas D. Potter \\ School of Psychology, University of Dundee, Dundee DD1 4HN, Scotland \\ Correspondence should be addressed to Douglas D. Potter, d.d.potter@dundee.ac.uk
}

Received 1 September 2010; Accepted 3 November 2010

Academic Editor: Tricia Striano

Copyright ( 92011 S. Webster and D. D. Potter. This is an open access article distributed under the Creative Commons Attribution License, which permits unrestricted use, distribution, and reproduction in any medium, provided the original work is properly cited.

\begin{abstract}
The Mindblindness model is the main model of social cognitive development in autism. This model assumes that eye direction detection and eye contact detection develop typically in autism (Baron-Cohen, 1995). The model's assumption of maturational development implies that when these skills are abnormal, they must either be absent or developmentally delayed. In contrast, the atypical modularisation hypothesis predicts that these skills can develop deviantly—successfully but atypically-in children with autism. Two computer-based tasks were used to assess eye direction detection and eye contact detection in children with autism and in typically developing children. These skills were developmentally deviant in children with autism. The findings support a model of social cognition in autism that accounts for developmental processes.
\end{abstract}

\section{Introduction}

Children with autism may attend to other people's eyes abnormally. For example, an absence of gaze following is a specific (but insensitive) marker for autism in infancy $[1,2]$. However, many children with autism do develop an ability to respond to gaze $[3,4]$. Gaze information is important in language development [5]. It may also be important for Theory of Mind development [6], although there is no strong evidence for this claim [7-9]. This study examined how gaze perception develops in children with autism.

The Mindblindness model is the main model of social cognitive development in autism $[6,10]$. Gaze perception is an important feature of that model. One of the modules ("neurocognitive mechanisms") in this model is the EyeDirection Detector (EDD). This mechanism is assumed to be "intact" (typical) in all children with autism [6]. EDD would detect the presence of eyes and would build dyadic representations of their direction. Two studies have examined eye direction detection in autism and have found this skill to be typical $[11,12]$. However, these studies did not examine reaction time data and did not examine the development of this skill. A more recent study, which measured reaction times and which used demanding stimuli, found deficits in eye direction detection in autism [13].
Eye contact detection is not typical in autism. A study of home videos made at the age of 12 months found that infants with autism looked less at other people than did developmentally delayed and typically developing infants [14]. A semistructured interview, administered to parents of children with autism, revealed abnormalities in the frequency and intensity of eye contact before 2 years of age [15]. Insensitivity to eye contact is apparent in experimental studies of children with autism. Gepner found that a group of children with autism and learning disability was less accurate at detecting eye contact than were younger, typically developing control groups [16]. Two recent studies found that children with autism (without learning disability) were less accurate than typically developing controls at detecting eye contact $[13,17]$. These studies did not examine the development of eye contact detection in autism.

In the Mindblindness model, EDD is a genetically prespecified mechanism that matures during development. Maturation of EDD implies that gaze perception in autism must either be "intact" (typical) or "impaired" (absent or developmentally delayed). The Mindblindness model does not allow for the possibility that gaze perception would develop deviantly (atypically) in autism. However, it is possible that eye direction detection and eye contact detection do develop in autism, but in a way that is specific to children 
with autism. This developmental pattern is predicted by the atypical modularisation hypothesis: that gaze processing develops successfully but atypically in children with autism. This hypothesis is central to our neuroconstructivist model of the development of gaze following in autism [18].

In autism, perception of the eyes in the context of a face is atypical. Children with autism demonstrate an advantage in face recognition when only the mouth or lower-face features are presented [19] whereas typical children benefit more from the eyes [20]. Children with autism benefit more from the lower half of the face than from the upper face when making judgements about emotional expressions, in contrast to typical children [21]. Similar findings have been made for adults with autism [22]. When watching actors on screen, adults with autism look more at the mouth and less at the eyes than do typical adults [23]. This bias towards looking at the mouth is unlikely to be a developmental delay: from the age of 7 weeks, infants will fixate more on the eye-region of the face when an adult begins to talk [24]. Together, these findings suggest that gaze perception is developmentally deviant rather than developmentally delayed in autism.

Two experiments were carried out to investigate whether gaze perception is typical, absent, developmentally delayed, or developmentally deviant in children with autism. Developmental effects were investigated by splitting participant groups into "younger" and "older" age groups. An autism group and a typical control group took part in these experiments. If the autism group had equivalent accuracy scores and reaction times to the control group, then gaze perception would be typical in the autism group. If the autism group performed at chance on both tasks, then eye direction detection and eye contact detection would be absent in that group. If the accuracy scores and reaction times of the older autism group resembled those of the younger typical control group, then gaze perception would be developmentally delayed in the older autism group. However, if the autism group scored above chance and produced atypical reaction times, then gaze perception would be developmentally deviant in these children with autism.

\section{Experiment 1: Eye Direction Detection}

This experiment tested one function of EDD: detecting the direction of another person's gaze. This task was based on a study reported by Leekam and colleagues [12]. The task used photographic images of a man looking at one of three coloured poles, with various distances between these poles and with eye and head direction either concordant or discordant (see [12]). Leekam found no significant differences in accuracy between autism and control groups on this task [12]. However, several of the participants in that study used a strategy of tracing an imaginary line on the stimuli with their finger, from the man's eyes to the location of the pole. Participants in our study held a gamepad that prevented them from using such a strategy. The gamepad also allowed measurement of reaction times. A detailed analysis of participants' accuracy scores and reaction times was carried out in the present study. Leekam's study used the 18 easiest stimuli from the original set [25], whereas this study used the full set of stimuli (36 images). This analysis included the participant's age group ("younger" or "older" group) as a factor to allow investigation of developmental effects. Leekam's group of participants with autism had much lower MA than CA and were learning disabled. Participants in our study did not have learning disability, which allowed us to control for cognitive ability level and chronological age with just one control group. The Autism Screening Questionnaire (ASQ: Berument et al. [26]) was used in our study to provide screening of participants in both groups.

\subsection{Method}

2.1.1. Participants. Participants were pupils at three primary schools and two secondary schools in and around Glasgow and at one primary school in Dundee. Participants in the autism group all attended communication disorder units within mainstream schools. Thirty children with autism and twenty-eight typically developing control participants took part in this experiment. Participants were included in the analysis only if they obtained a scaled score of 8 or above on the Vocabulary subscale of the WISC-R and a prorated IQ of 85 or above based on Vocabulary and Block Design scaled scores. Participants who were included in the analysis all had ASQ scores within the appropriate range for their participant group (15 or above for autism, below 15 for controls) [26].

All children in the autism group had previously received clinical diagnoses of autism, autism spectrum disorder, or Asperger syndrome. Data from 16 children in this group had to be excluded. 7 participants had prorated IQs below 85 and were dropped from the analysis. One autism group participant had to be dropped to allow between-group matching on Vocabulary scaled score. This participant had scored 18 on a 19-point scale. The parents of 4 participants did not return the ASQ and 2 participants each scored 10 on the ASQ, below the ASQ's 15-point cut-off for autism. Two participants were excluded from the autism group due to diagnoses of dyspraxia, a disorder of motor control. These participants were excluded because reaction times were an important measure in this study.

None of the children in the control group had a diagnosis of autism, autism spectrum disorder, or Asperger syndrome. Parents of 4 children in this group failed to return the ASQ and these four children were excluded from the analysis. In total, data from 14 children with autism and 24 typically developing children was analysed. For analysis, the autism group consisted of 13 males and 1 female. The control group consisted of 19 males and 5 females. The autism and control groups were each split into equal-sized younger and older groups to allow the use of "age group" as a factor.

The autism group and control group were closely matched on age, WISC-R Vocabulary scaled score, WISC$\mathrm{R}$ Block Design scaled score, and prorated IQ. Vocabulary and Block Design were chosen because these two WISC$\mathrm{R}$ subtests have the highest correlation with Full Scale IQ for any verbal and nonverbal dyad (Sattler [27, page 851]: 
TABLE 1: Group matching data for the autism group and control group (experiment 1). Values are mean values \pm SD.

\begin{tabular}{lcccc}
\hline Participant group & Age & Vocabulary scaled score & Block design scaled score & Prorated IQ \\
\hline Autism $(n=14)$ & $11.2 \pm 3.5$ & $10.4 \pm 2.0$ & $12.0 \pm 3.1$ & $107.5 \pm 11.7$ \\
Control $(n=24)$ & $10.6 \pm 3.2$ & $10.8 \pm 2.5$ & $11.5 \pm 3.1$ & $106.7 \pm 12.7$ \\
\hline
\end{tabular}

TABLE 2: Matching data for the younger and older autism and control groups (experiment 1). Values are mean values \pm SD.

\begin{tabular}{lcccc}
\hline Participant group & Age & Vocabulary scaled score & Block design scaled score & Prorated IQ \\
\hline Younger autism $(n=7)$ & $8.4 \pm 0.9$ & $10.1 \pm 2.0$ & $11.3 \pm 2.1$ & $104.3 \pm 7.3$ \\
Younger controls $(n=12)$ & $7.8 \pm 1.3$ & $11.3 \pm 2.3$ & $10.2 \pm 2.6$ & $104.3 \pm 10.9$ \\
Older autism $(n=7)$ & $14.0 \pm 2.7$ & $10.7 \pm 2.2$ & $12.7 \pm 3.9$ & $110.7 \pm 14.8$ \\
Older controls $(n=12)$ & $13.4 \pm 1.8$ & $10.3 \pm 2.6$ & $12.8 \pm 3.1$ & $109.0 \pm 14.4$ \\
\hline
\end{tabular}

$r=.91$ for WISC-R). Table 1 summarises the participant group characteristics. Independent samples $t$-tests (2-tailed) were carried out on the matching data. Groups were closely matched $(P>.50$ for all variables: see $[28])$ on measures of age $(t(36)=.53, P=.60)$, Vocabulary scaled score $(t(36)=$ $-.52, P=.61)$, Block Design scaled score $(t(36)=.52$, $P=.61)$, and prorated IQ $(t(36)=.76, P=.84)$.

Groups were split according to age (see Table 2). The younger autism group and younger control group were closely matched on prorated IQ $(t(17)=-.01, P=.99)$. Groups were not closely matched on age $(t(17)=1.05$, $P=.31)$, Vocabulary scaled score $(t(17)=-1.14, P=.27)$ or Block Design scaled score $(t(17)=.97, P=.35)$, but there were no significant differences between the groups on these variables. The older autism and control groups were closely matched $(P>.50)$ on all variables: on age $(t(17)=.57$, $P=.56)$, Vocabulary scaled score $(t(17)=.32, P=.75)$, Block Design scaled score $(t(17)=-.02, P=.98)$ and prorated IQ $(t(17)=.25, P=.81)$.

2.1.2. Apparatus. The experiment was run with DMDX experimental software, developed at Monash University and at the University of Arizona by K. I. Forster and J. C. Forster. DMDX ran on an IPC laptop computer (Pentium III processor, $1 \mathrm{GHz})$ attached to a 15.8 -inch $(40 \mathrm{~cm})$ colour monitor. Participants sat about $65 \mathrm{~cm}$ from this monitor. Participants responded using a PC gamepad. This was attached to the laptop using a USB port and was interrupt driven. Error rates and reaction times were measured from these responses, which were recorded to within the nearest millisecond.

2.1.3. Stimuli. Stimuli were scanned digital images of colour photographs. The original photographs were taken using a $35 \mathrm{~mm}$ camera with a $90 \mathrm{~mm}$ lens. Each image consisted of a man (on the viewer's right) looking at one of three coloured poles (to the viewer's left). From left, these poles always appeared in the same order: red, yellow, and blue. The poles were $45 \mathrm{~mm}$ high in real life. There were 36 images in total. There were four sets of 9 images, and the coloured poles were $20,15,10$, and 5 degrees apart in each of these sets of images. These angles were measured relative to the man's head, which was positioned $53 \mathrm{~cm}$ away from the rods and $280 \mathrm{~cm}$ from the camera during generation of the original stimulus set. The directions of both the head and the eyes were varied systematically. In one-third of the images, the head and eyes were concordant (pointing in the same direction). In the other two-thirds of the images, head and eye directions were discordant. Participants were therefore unable to work out where the man in the image was looking based on head direction alone.

2.1.4. Design. The task consisted of 37 trials. One practice trial was followed by 36 test trials. Trials were presented in four blocks, and the order of these blocks was fixed: 20, 15, 10 , then 5 degrees between poles. The order of trials within each block was randomised. There was one within group factor (angle between poles) with four levels.

2.1.5. Procedure. Each child was assessed within their own school, either in a quiet room or in a quiet space within their usual classroom. Participants were asked to identify the red, yellow, and blue poles. They were then asked to work out which pole the man in the photograph was looking at and to press the button on the gamepad that was the same colour as that pole. There was no time limit on responses. Participants were also asked not to touch the screen, to prevent them from using the strategy of tracing a line from the stimulus' eyes to the poles [12].

2.2. Results. All detection responses were included but reaction times less than $100 \mathrm{~ms}$ or greater than $5000 \mathrm{~ms}$ were excluded from analyses. Mean accuracy scores for all participants are presented in Table 3. Mean reaction times for all participants are presented in Table 4.

In the analysis of accuracy scores, main effects of participant group, angle and age group were significant. The main effect of participant group $(F[1,34]=3.424$, $P=.003$ ) arose because the autism group was less accurate than the control group. Independent samples $t$-tests (2tailed) demonstrated that the autism group was significantly less accurate than the control group on 20 degrees stimuli $(t(36)=-2.68, P=.01)$ and on 10 degrees stimuli $(t(36)=-2.79, P=.02)$. The autism and control groups 
TABLE 3: Mean scores of the autism group and control group in the eye direction detection task (experiment 1).

\begin{tabular}{|c|c|c|c|c|}
\hline Angle between poles (Mean score \pm SD) & 20 degrees & 15 degrees & 10 degrees & 5 degrees \\
\hline Autism & $6.5 \pm 2.0$ & $6.3 \pm 2.4$ & $4.8 \pm 2.4$ & $4.2 \pm 1.9$ \\
\hline Controls & $8.0 \pm 1.3$ & $7.3 \pm 1.6$ & $6.6 \pm 1.7$ & $5.1 \pm 2.1$ \\
\hline
\end{tabular}

TABLE 4: Mean reaction times of the autism group and control group in the eye direction detection task (experiment 1), in milliseconds.

\begin{tabular}{lcccc}
\hline Angle between poles $($ Mean RT \pm SD) & 20 degrees & 15 degrees & 10 degrees & 5 degrees \\
\hline Autism & $2048 \pm 730$ & $1699 \pm 619$ & $1904 \pm 561$ & $1770 \pm 554$ \\
Controls & $2521 \pm 1350$ & $2202 \pm 961$ & $2518 \pm 1180$ & $2710 \pm 857$ \\
\hline
\end{tabular}

TABLE 5: Mean total accuracy scores for the autism group and control group, by age group, in the eye direction detection task (mean scores $\pm \mathrm{SD}$ ).

\begin{tabular}{lcc}
\hline \multirow{2}{*}{ Participant group } & \multicolumn{2}{c}{ Age group } \\
& Younger & Older \\
\hline Autism & $16.7 \pm 5.1$ & $26.9 \pm 4.6$ \\
Control & $24.8 \pm 4.9$ & $29.0 \pm 4.7$ \\
\hline
\end{tabular}

performed above chance in all conditions, with an average score above 3 (out of 9) in all conditions. The main effect of angle $(F[3,102]=24.783, P<.001)$ was due to participants' accuracy decreasing as the angle between poles in the stimulus image decreased. The interaction between participant group and angle was not significant $(F[3,108]=$ $.96, P=.42)$.

The main effect of age group was significant because older participants were more accurate than younger participants $(F[1,34]=19.631, P<.001)$. The interaction between participant group (autism, control) and age group (younger, older) was marginally significant $(F[1,34]=3.424, P=$ $.07)$. A Tukey HSD test revealed that the accuracy score of the younger autism group was significantly lower than the mean score of the other three groups (older autism, younger control, and older control groups). $t$-tests revealed that the younger autism group was significantly less accurate than the younger control group for 20 degree stimuli $(t(17)=$ $-2.126, P=.05)$ and for 10 degree stimuli $(t(17)=-1.583$, $P<.01)$. For 15-degree stimuli, the effect of accuracy was marginally significant $(t(17)=-1.936, P=.07)$, with the younger autism group being less accurate than the younger control group. The older autism group was less accurate than the older control group on 20 degree stimuli only $(t(17)=$ $-2.147, P=.05$ ) (see Table 5).

In the analysis of reaction times, the main effect of participant group was significant $(F[1,34]=5.556, P=.03)$. The autism group was faster to respond than the control group. $t$-tests revealed that the autism group was significantly faster than the control group on 5-degree stimuli $(t(36)=$ $-3.668, P=.001)$. There were trends towards the autism group being faster than the control group on 10-degree stimuli $(t(36)=-1.822, P=.08)$ and on 15-degree stimuli $(t(36)=-1.752, P=.09)$. There was a marginally significant main effect of angle $(F[3,102]=2.405, P=.07)$. There was no main effect of age group $(F[1,34]=.439, P=.51)$. However, there was a significant interaction between angle and age group $(F[3,102]=3.414, P=.02)$ (see Table 6 ). Younger participants were slower than older participants on 20-degree stimuli, but this effect was only marginally significant $(t(36)=1.919, P=.06)$.

2.3. Discussion. There was a deficit in eye direction detection accuracy for the autism group on this task. The deficit was not due to developmental delay in this function, as the older autism group did not perform the task in the same way as younger controls. Eye direction detection was not absent in the autism group as this group performed above chance. As for the control group, the autism group's performance decreased with increasing task difficulty (the main effect of "angle"). However, the autism group responded more rapidly than the control group. Eye direction detection was developmentally deviant-successful but atypical-in these children with autism.

Developmental effects were also evident. Older participants were more accurate than younger participants. Analysis also revealed that the extent of the eye direction detection deficit in autism decreases over time. The younger autism group was less accurate than the younger control group for 10-degree and 20-degree stimuli and was marginally less accurate for 15-degree stimuli. However, the older autism group was only less accurate than the older control group on 20-degree stimuli, the easiest stimulus set. The deficit in eye direction detection appears to ameliorate with increasing age.

Children with autism were less accurate than typical children at detecting the direction of gaze on the easiest stimuli in this task. Typical children may have more experience of detecting gaze direction, and the discrimination of small angles of gaze (such as 5 degrees) may be less common than the discrimination of gaze direction at large visual angles [29]. Typical children may therefore have the most experience of eye direction detection for large visual angles of around 20 degrees rather than for fine visual angles of around 5 degrees. Alternatively, the accuracy scores for this task may reflect a combination of two effects. Both groups may have had low accuracy scores on trials with small visual angles simply because of the high visual spatial processing demands of these trials. For typical children, this factor may have combined with their extensive experience of eye direction 
TABLE 6: Mean reaction times (in milliseconds \pm SD) for stimuli at each angle, by age group, in the eye direction detection task (experiment $1)$.

\begin{tabular}{|c|c|c|c|c|}
\hline \multirow{2}{*}{ Age group } & \multicolumn{4}{|c|}{ Angle } \\
\hline & 20 degrees & 15 degrees & 10 degrees & 5 degrees \\
\hline Younger $(n=19)$ & $2699 \pm 1448$ & $2209 \pm 907$ & $2395 \pm 1318$ & $2237 \pm 877$ \\
\hline Older $(n=19)$ & $1994 \pm 683$ & $1824 \pm 825$ & $2188 \pm 658$ & $2490 \pm 889$ \\
\hline
\end{tabular}

detection to give significantly higher accuracy scores on trials with larger visual angles, but equivalent accuracy scores in the autism and control groups for more difficult trials.

Using this task, Leekam found no significant differences in accuracy between a group of children with autism and two control groups (one group of typically developing children and one group of learning disabled children: Leekam et al. [12]). In fact, there was a trend towards superior performance in the autism group. In Leekam's study, the autism group (mean age 13.4 years) was much older than both control groups (typically developing: 4.3 years, learning disabled: 6.2 years). This discrepancy was a consequence of group matching on mental age. In the present study, there was no such age discrepancy, and this study may therefore provide clearer insights into developmental effects.

Leekam observed that on this task, using real photographs, some children with autism used a visual-spatial strategy of drawing an imaginary line on the stimuli [12]. In the present study, the administration of this task on computer prevented the use of such a strategy. This difference in method may explain, to some extent, why deficits in the autism group's performance were observed in this study but not in Leekam's study. The children with autism in this study may have used visual spatial processing to support their performance, evidently with some success. However, typical children would have been able to apply their extensive experience of detecting gaze direction in this task, in addition to their visual spatial skills.

\section{Experiment 2: Eye Contact Detection}

This experiment tested another function of EDD: detecting eye contact. Like the eye direction detection task, reported above, this task was designed for the study of eye gaze perception in prosopagnosia [30]. The task has previously been administered to a group of adults and older children with autism [31] and to children with autism and learning disability [16]. Howard found that adults and older children with autism were less accurate on this task than typically developed controls [31].

As for Leekam's eye direction detection study, Gepner's study involved children with autism and learning disability (mean age 11.3 years) and therefore required several control groups: typical controls matched on verbal ability (mean age 5.6 years), typical controls matched on nonverbal ability (mean age 5.9), and learning-disabled controls with Down syndrome (mean age 15.9 years) [16]. Gepner found that children with autism were less accurate than typical children and Down syndrome children on this task. As for the replication of Leekam's eye direction detection study, the present study has several advantages. In particular, the present study required only one control group (matched on age, verbal ability, nonverbal ability, and prorated IQ), reaction times were measured, and the Autism Screening Questionnaire was used for participant screening. In order to assess developmental effects, age group was used as a factor in this study.

\subsection{Methods}

3.1.1. Participants and Apparatus. The same participants who took part in the eye direction detection task took part in this task. The order of administration of the eye direction detection and eye contact detection tasks was randomised. Data from the same participants was analysed and the same group-matching criteria were used. For this experiment, testing conditions and apparatus were the same as for the eye direction detection task, above.

3.1.2. Stimuli. Stimuli were scanned digital images of colour photographs. Each image was of a man looking either towards or away from the viewer's eyes. There were 21 images in total: 3 images with direct gaze and 18 images with averted gaze. In these images, the man's head was either directed towards the viewer or was directed 20 degrees to the left or right. The man's eyes were either focussed on the viewer's eyes (zero degrees from midline) or at a point 5, 10, or 20 degrees to the left or right. There were no targets or objects in the images.

3.1.3. Design. The task consisted of 37 trials, with one practice trial and 36 test trials. On each trial, one eye-contact image was presented on the computer monitor alongside one image with eyes averted by 5,10 , or 20 degrees. One image was presented on the left of the screen and the other on the right of the screen. For each trial, the direction of the head was the same in both images. There were 3 blocks of 12 trials. Images with eyes averted by 20 degrees were presented in the first block. The second block included images with eyes averted by 10 degrees, and the third block included images with eyes averted by 5 degrees. Within each block, each pair of stimuli (eye contact and eyes averted) was presented twice. On the second presentation of each pair, the on-screen position of the images (left or right) was reversed. The order of presentation of pairs of images was randomised within blocks.

3.1.4. Procedure. Participants were informed that they would see a fixation cross in the centre of the screen. They were 
TABLE 7: Accuracy data for the participant group by age group interaction in the eye contact detection task (experiment 2). Values are total scores for each subgroup $\pm \mathrm{SD}$.

\begin{tabular}{lcc}
\hline \multirow{2}{*}{ Participant group } & \multicolumn{2}{c}{ Age group } \\
& Younger & Older \\
\hline Autism & $25.7 \pm 3.1$ & $28.8 \pm 3.5$ \\
Controls & $31.1 \pm 1.5$ & $29.8 \pm 2.1$ \\
\hline
\end{tabular}

TABLE 8: Reaction time data for the eye contact detection task (experiment 2) for the age group by angle interaction. Values are mean RTs \pm SD in milliseconds.

\begin{tabular}{lccc}
\hline Age group & \multicolumn{3}{c}{ Angle } \\
& 20 degrees & 10 degrees & 5 degrees \\
\hline Younger $(n=19)$ & $1990 \pm 309$ & $2149 \pm 621$ & $2487 \pm 561$ \\
Older $(n=19)$ & $1377 \pm 301$ & $1680 \pm 474$ & $2307 \pm 527$ \\
\hline
\end{tabular}

TABle 9: Reaction time data for the eye contact detection task (experiment 2) for the participant group by angle interaction. Values are mean RTs \pm SD in milliseconds.

\begin{tabular}{lccc}
\hline Participant group & \multicolumn{3}{c}{ Angle } \\
& 20 degrees & 10 degrees & 5 degrees \\
\hline Autism $(n=14)$ & $1721 \pm 300$ & $1921 \pm 724$ & $2151 \pm 316$ \\
Controls $(n=24)$ & $1662 \pm 499$ & $1911 \pm 522$ & $2541 \pm 601$ \\
\hline
\end{tabular}

instructed to look at this cross and were told that it would be followed by two pictures of a man's face. These steps were demonstrated. The participant was told that in one of the pictures, the man would be looking towards the participant. When the eye contact image was on the left of the screen, participants pressed a button on the left of the gamepad. When this image was on the right, they pressed a button on the right. There was no time limit on responses.

3.2. Results. In the analysis of response errors, the main effect of participant group was not significant $(F[1,34]=.922$, $P=.34$ ). Both participant groups performed above chance ( 6 out of 12) with a mean score of at least 8 in all conditions. Main effects of age group and of angle were significant (age group: $(F[1,34]=11.424, P=.002)$, angle: $(F[1,34]=$ $45.563, P<.01)$ ). Younger participants were less accurate than older participants, and all participant groups performed worse on more difficult stimuli (as angle of gaze aversion decreased). There was a significant interaction between participant group and age group $(F[1,34]=5.311, P=.03)$. Tukey's HSD test $(\alpha<.05)$ revealed that the younger autism group was as accurate as the older autism group but was significantly less accurate than both the younger and older control groups (see Table 7).

In the analysis of reaction times, the main effect of participant group was not significant $(F[1,34]=.919, P=$ $.35)$. The main effect of angle was significant $(F[2,68]=$ 22.83, $P<.01)$. All participant groups responded more slowly to more difficult stimuli. The main effect of age group was also significant $(F[1,24]=11.536, P=.002)$.
Younger participants were slower to respond than were older participants. The interaction between angle and age group approached significance $(F[2,68]=2.759, P=.07)$. Independent samples $t$-tests (2-tailed) revealed that younger participants were significantly slower than older participants in responding to 10 -degree stimuli $(t(36)=2.620, P=.01)$ and 20-degree stimuli $(t(36)=6.197, P<.01$ ) only (see Table 8).

There was also a significant interaction between angle and participant group $(F[2,68]=3.127, P=.05)$ (see Table 9). Tukey HSD tests $(\alpha<.05)$ revealed that the autism group was faster to respond to 20-degree stimuli than to 5-degree stimuli, and that the control group was faster to respond to 20-degree and 10-degree stimuli than to 5 -degree stimuli. The autism group was significantly faster than the control group when responding to 5 degree stimuli $(t(35.8)=-2.614, P=.01)$, although there was no group difference in accuracy for these stimuli $(t(36)=-.570, P=$ $.57)$.

This experiment revealed a developmental effect in eye contact detection for children with autism. There was a significant interaction between participant group and age group. A posthoc test revealed that the younger autism group was as accurate as the older autism group but was significantly less accurate than younger and older control groups. The older autism group performed as well as both control groups, and there was no difference in accuracy between the younger and older control groups. Together, these findings suggest that children with autism are poor at eye contact detection in early childhood but become as proficient as typically-developing children in later childhood. The findings could be interpreted as demonstrating either developmental delay or developmental deviance. The analysis of reaction time data, discussed below, suggests that eye contact detection in autism is deviant rather than delayed.

For reaction times, there was a significant interaction between angle and participant group. The autism group responded faster than the control group on the most difficult stimuli (5 degrees). Both groups responded more quickly on those trials where one stimulus was clearly not making eye contact (e.g., 20-degree trials) than on trials where both stimuli could appear to be looking towards the viewer (5-degree trials). This pattern of performance was more pronounced in the control group, which was also faster to respond on 10-degree trials than on 5-degree trials. Trials with 5-degree stimuli may have taken longer to resolve because these stimuli can appear to be looking at the viewer.

In typical development, direct gaze is detected more rapidly and accurately than averted gaze [32] and direct gaze speeds up the process of face categorisation [33]. This processing advantage for direct gaze may have a motivational component. In typical development, enhanced, rapid processing of images of faces with direct eye contact could arise due to extensive experience of such stimuli as being motivating and reinforcing: face stimuli with direct gaze are worth attending to. In the present study, controls were slower than participants with autism when discriminating 
direct gaze from gaze averted by 5 degrees. In that condition, both images look towards the viewer (even though one image is not making eye contact). Both images are therefore highly salient to typical viewers, who may be unable to distinguish the stimuli solely on the basis of motivational salience.

The autism group was as accurate as controls at detecting all stimuli and was at least as rapid as controls on all trials. Children with autism may be insensitive to the reinforcement value of social stimuli including gaze signals $[34,35]$. In the present task, reduced salience of direct gaze could have led to the reaction time advantage in the autism group for 5degree stimuli, relative to the control group. The pattern of reaction times in the present task may reflect motivational effects of direct gaze that are specific to the control group. In contrast to typical children, children with autism may only use visual spatial processing in the performance of this eye contact detection task.

\section{General Discussion}

This study tested the main model of gaze perception development in autism, the Mindblindness model [6]. Based on the supposition of intact and impaired social cognitive functions in autism, that model proposes that there are several discrete modules for social cognition in typically developing children and adults. In particular, the model assumes that eye direction detection and eye contact detection, functions of EDD, are "intact" in autism. This study demonstrates that EDD is not "intact" in autism. Children with autism were less accurate at eye direction detection than were typical children. In the eye contact detection task, younger participants with autism were less accurate than were younger and older typical children. On their own, these accuracy findings could be interpreted as demonstrating developmental delay in the maturation of EDD. EDD's eye direction detection function could be impaired and EDD's eye contact detection function could be developmentally delayed until later childhood or adolescence. Such an interpretation would suggest that EDD's definition within the Mindblindness model was essentially correct and that all that was required was a more careful delineation of EDD's maturation in autism.

The analyses of reaction time data from these two tasks contradict that interpretation. On the eye direction detection task, participants with autism were faster to respond than were control participants. Although participants with autism were less accurate than controls, the autism group responded above chance in all conditions. Eye direction detection was present in the autism group but was performed atypically: less accurately and more rapidly. In the eye contact detection task, the autism group was significantly faster than the control group when responding on the most difficult trials and was as accurate as the control group on all trial types. These skills were not "intact" but neither were they "impaired". It would be inaccurate to characterise these skills as developmentally delayed in autism. Older children with autism did not perform these skills in the same way as younger, typically developing children. These tasks may instead demonstrate autism-specific patterns of performance on eye direction detection and eye contact detection that do not resemble the patterns of performance which emerge in typical development.

Reaction times for children with autism were faster than those for typically developing children in all conditions of the eye direction detection task, and in the most difficult condition of the eye contact detection task. Typical children attend to social stimuli for longer than do children with autism [36]. Reduced attention to social stimuli, particularly the eyes [23], could explain why faster reaction times were observed in the autism group. These tasks may not be "social" tasks for children with autism. These children may approach the tasks in the same way that they would approach a nonsocial visual spatial task, and the reaction time advantage for the autism group may reflect superior visual-spatial processing in this group [37-40]. As discussed above, a motivational bias to attend to direct gaze may have caused typical control participants to respond more slowly than children with autism on the most difficult trials in the eye contact detection task. A lack of attention to eyes would also explain the pattern of accuracy scores and reaction times for children with autism on the eye contact task. The autism group was faster than controls to respond to the most difficult stimuli and was no less accurate than controls on these trials.

The findings are consistent with a developmental, neuroconstructivist approach to developmental disorders [41] including autism [18]. The autism group and the control group may have used different cognitive mechanisms to perform this task. In adulthood, functions such as eye direction detection and eye contact detection may depend on domain-specific cognitive modules [42]. However, such modules may develop differently in developmental disorders $[41,43]$. The present findings indicate that children with autism may lack a typical motivational bias to attend preferentially to other people's eyes, consistent with previous findings which suggest that children with autism attend less to the eyes than do typical children $[19,20,23]$. In these children with autism, specialised cognitive mechanisms for gaze processing may have developed successfully but atypically.

In infancy, gaze behaviour occurs within a strongly motivating affective context [44]. Dawson's research suggests that gaze processing develops abnormally in autism because children with autism are relatively insensitive to the reinforcement value of social stimuli $[35,45]$. In contrast, typically developing children may learn to prioritise social information over nonsocial information through their experience of social interactions [46]. In autism, a failure to prioritise social information would disrupt the modularisation of gaze perception, leading to successful but atypical development of cognitive modules for eye direction detection and eye contact detection in autism.

\section{Abbreviations}

ASQ: Autism Screening Questionnaire

EDD: Eye-Direction Detector. 


\section{Acknowledgments}

This research was funded by the Robertson Trust and the Hugh Fraser Foundation. The authors thank Professor Dave Perrett for providing them with the stimuli that were used in this study. They also thank school pupils (and teachers) who helped with this research at Blackness Primary School, Dundee (Roberta Russell); Carlibar Primary School, Barrhead (Moira Park); Crosshouse Primary School, East Kilbride (Kitty Welsby); Cumbernauld High School, Cumbernauld (Ros Palmer, Mark Cairns, and Bridget Raeside); Hillpark Secondary School, Glasgow (Nadine Barber); and St Vincent's CDU, Glasgow (Isobel McAllister). Irene Grant at Glasgow City Council helped with the selection of schools.

\section{References}

[1] G. Baird, T. Charman, S. Baron-Cohen et al., "A screening instrument for autism at 18 months of age: a 6-year followup study," Journal of the American Academy of Child and Adolescent Psychiatry, vol. 39, no. 6, pp. 694-702, 2000.

[2] S. Baron-Cohen, A. Cox, G. Baird et al., "Psychological markers in the detection of autism in infancy in a large population," British Journal of Psychiatry, vol. 168, pp. 158163, 1996.

[3] S. R. Leekam, E. Hunnisett, and C. Moore, "Targets and cues: gaze-following in children with autism," Journal of Child Psychology and Psychiatry and Allied Disciplines, vol. 39, no. 7, pp. 951-962, 1998.

[4] S. R. Leekam, B. López, and C. Moore, "Attention and joint attention in preschool children with autism," Developmental Psychology, vol. 36, no. 2, pp. 261-273, 2000.

[5] M. Morales, P. Mundy, C. E. F. Delgado et al., "Responding to joint attention across the 6- through 24-month age period and early language acquisition," Journal of Applied Developmental Psychology, vol. 21, no. 3, pp. 283-298, 2000.

[6] S. Baron-Cohen, Mindblindness: An Essay on Autism and Theory of Mind, MIT Press, Boston, Mass, USA, 1995.

[7] M. J. Doherty and J. R. Anderson, "A new look at gaze: preschool children's understanding of eye-direction," Cognitive Development, vol. 14, no. 4, pp. 549-571, 1999.

[8] K. Lee, M. Eskritt, L. A. Symons, and D. Muir, "Children's use of triadic eye gaze information for "mind reading", Developmental psychology, vol. 34, no. 3, pp. 525-539, 1998.

[9] E. Pellicano and G. Rhodes, "The role of eye-gaze in understanding other minds," British Journal of Developmental Psychology, vol. 21, no. 1, pp. 33-43, 2003.

[10] S. Baron-Cohen, "How to build a baby that can read minds-cognitive mechanisms in mindreading," Cahiers De Psychologie Cognitive-Current Psychology of Cognition, vol. 13, no. 5, pp. 513-552, 1994.

[11] S. Baron-Cohen, "Perceptual role-taking and protodeclarative pointing in autism," British Journal of Developmental Psychology, vol. 7, pp. 113-127, 1989.

[12] S. Leekam, S. Baron-Cohen, D. Perrett, M. Milders, and S. Brown, "Eye-direction detection: a dissociation between geometric and joint attention skills in autism," British Journal of Developmental Psychology, vol. 15, no. 9981, pp. 77-95, 1997.

[13] J. Swettenham, K. Plaisted, E. Milne, R. Campbell, D. Rosenfelder, and M. Coleman, "Children with autism show subtle difficulties detecting eye direction," in Proceedings of
theAnnual Meeting of the Cognitive Neuroscience Society, New York, NY, USA, 2001.

[14] J. A. Osterling, G. Dawson, and J. A. Munson, "Early recognition of 1-year-old infants with autism spectrum disorder versus mental retardation," Development and Psychopathology, vol. 14, no. 2, pp. 239-251, 2002.

[15] D. C. Wimpory, R. P. Hobson, J. M. G. Williams, and S. Nash, "Are infants with autism socially engaged? A study of recent retrospective parental reports," Journal of Autism and Developmental Disorders, vol. 30, no. 6, pp. 525-536, 2000.

[16] B. Gepner, B. de Gelder, and S. de Schonen, "Face processing in autistics: evidence for a generalised deficit?" Child Neuropsychology, vol. 2, no. 2, pp. 123-139, 1996.

[17] A. Senju, K. Yaguchi, Y. Tojo, and T. Hasegawa, "Eye contact does not facilitate detection in children with autism," Cognition, vol. 89, no. 1, pp. B43-B51, 2003.

[18] S. Webster, The development of gaze following in autism, thesis, University of Dundee, 2004, Unpublished Thesis.

[19] T. Langdell, "Recognition of faces: an approach to the study of autism," Journal of Child Psychology and Psychiatry and Allied Disciplines, vol. 19, no. 3, pp. 255-268, 1978.

[20] R. M. Joseph and J. Tanaka, "Holistic and part-based face recognition in children with autism," Journal of Child Psychology and Psychiatry and Allied Disciplines, vol. 44, no. 4, pp. 529-542, 2003.

[21] T. F. Gross, "The perception of four basic emotions in human and nonhuman faces by children with autism and other developmental disabilities," Journal of Abnormal Child Psychology, vol. 32, no. 5, pp. 469-480, 2004.

[22] S. Baron-Cohen, S. Wheelwright, and T. Jolliffe, "Is there a "language of the eyes"? Evidence from normal adults, and adults with autism or Asperger Syndrome," Visual Cognition, vol. 4, no. 3, pp. 311-331, 1997.

[23] A. Klin, W. Jones, R. Schultz, F. Volkmar, and D. Cohen, "Visual fixation patterns during viewing of naturalistic social situations as predictors of social competence in individuals with autism," Archives of General Psychiatry, vol. 59, no. 9, pp. 809-816, 2002.

[24] M. M. Haith, T. Bergman, and M. J. Moore, "Eye contact and face scanning in early infancy," Science, vol. 198, no. 4319, pp. 853-855, 1977.

[25] D. Perrett and M. Milders, "Studies of gaze direction," Unpublished manuscript: Psychological Laboratory, Univeristy of St Andrews, 1992.

[26] S. K. Berument, M. Rutter, C. Lord, A. Pickles, and A. Bailey, "Autism screening questionnaire: diagnostic validity," British Journal of Psychiatry, vol. 175, pp. 444-451, 1999.

[27] J. M. Sattler, Assessment of Children, Jerome M. Sattler, San Diego, Calif, USA, 3rd edition, 1992.

[28] C. B. Mervis and B. P. Klein-Tasman, "Methodological issues in group-matching designs: $\alpha$ levels for control variable comparisons and measurement characteristics of control and target variables," Journal of Autism and Developmental Disorders, vol. 34, no. 1, pp. 7-17, 2004.

[29] S. Thayer, "Children's detection of on-face and off-face gazes," Developmental Psychology, vol. 13, no. 6, pp. 673-674, 1977.

[30] R. Campbell, C. A. Heywood, A. Cowey, M. Regard, and T. Landis, "Sensitivity to eye gaze in prosopagnosic patients and monkeys with superior temporal sulcus ablation," Neuropsychologia, vol. 28, no. 11, pp. 1123-1142, 1990.

[31] M. A. Howard, P. E. Cowell, J. Boucher et al., "Convergent neuroanatomical and behavioural evidence of an amygdala hypothesis of autism," NeuroReport, vol. 11, no. 13, pp. 2931$2935,2000$. 
[32] M. von Grünau and C. Anston, "The detection of gaze direction: a stare-in-the-crowd effect," Perception, vol. 24, no. 11, pp. 1297-1313, 1995.

[33] C. N. Macrae, B. M. Hood, A. B. Milne, A. C. Rowe, and M. F. Mason, "Are you looking at me? Eye gaze and person perception," Psychological Science, vol. 13, no. 5, pp. 460-464, 2002.

[34] G. Dawson, A. N. Meltzoff, J. Osterling, and J. Rinaldi, "Neuropsychological correlates of early symptoms of autism," Child Development, vol. 69, no. 5, pp. 1276-1285, 1998.

[35] G. Dawson, J. Munson, A. Estes et al., "Neurocognitive function and joint attention ability in young children with autism spectrum disorder versus developmental delay," Child Development, vol. 73, no. 2, pp. 345-358, 2002.

[36] J. Swettenham, S. Baron-Cohen, A. Cox et al., "The frequency and distribution of spontaneous attention shifts between social and nonsocial stimuli in autistic, typically developing, and nonautistic developmentally delayed infants," Journal of Child Psychology and Psychiatry and Allied Disciplines, vol. 39, no. 5, pp. 747-753, 1998.

[37] M. J. Caron, L. Mottron, C. Rainville, and S. Chouinard, "Do high functioning persons with autism present superior spatial abilities?” Neuropsychologia, vol. 42, no. 4, pp. 467-481, 2004.

[38] F. Happé, “Autism: cognitive deficit or cognitive style?" Trends in Cognitive Sciences, vol. 3, no. 6, pp. 216-222, 1999.

[39] M. A. O'Riordan, "Superior visual search in adults with autism," Autism, vol. 8, no. 3, pp. 229-248, 2004.

[40] M. A. O'Riordan, K. C. Plaisted, J. Driver, and S. Baron-Cohen, "Superior visual search in autism," Journal of Experimental Psychology: Human Perception and Performance, vol. 27, no. 3, pp. 719-730, 2001.

[41] A. Karmiloff-Smith, "Development itself is the key to understanding developmental disorders," Trends in Cognitive Sciences, vol. 2, no. 10, pp. 389-398, 1998.

[42] D. I. Perrett and N. J. Emery, "Understanding the intentions of others from visual signals-neurophysiological evidence," Cahiers De Psychologie Cognitive-Current Psychology of Cognition, vol. 13, no. 5, pp. 683-694, 1994.

[43] S. Webster and D. D. Potter, "Brief report: eye direction detection improves with development in autism," Journal of Autism and Developmental Disorders, vol. 38, no. 6, pp. 11841186, 2008.

[44] L. B. Adamson and C. L. Russell, "Emotion regulation and the emergence of joint attention," in Early Social Cognition: Understanding others in the First Months of Life, P. Rochat, Ed., Erlbaum, Mahwah, NJ, USA, 1999.

[45] G. Dawson, J. Osterling, J. Rinaldi, L. Carver, and J. McPartland, "Brief report: recognition memory and stimulus-reward associations: indirect support for the role of ventromedial prefrontal dysfunction in autism," Journal of Autism and Developmental Disorders, vol. 31, no. 3, pp. 337-341, 2001.

[46] S. R. Leekam, "Why do children with autism have a joint attention impairment?" in Joint Attention: Perspectives from Philosophy and Psychology, N. Eilan, C. Hoerl, T. McCormack, and J. Roessler, Eds., Oxford University Press, Oxford, UK, 2002. 


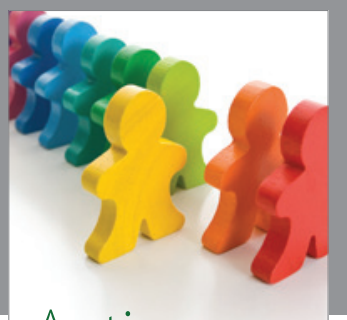

Autism

Research and Treatment
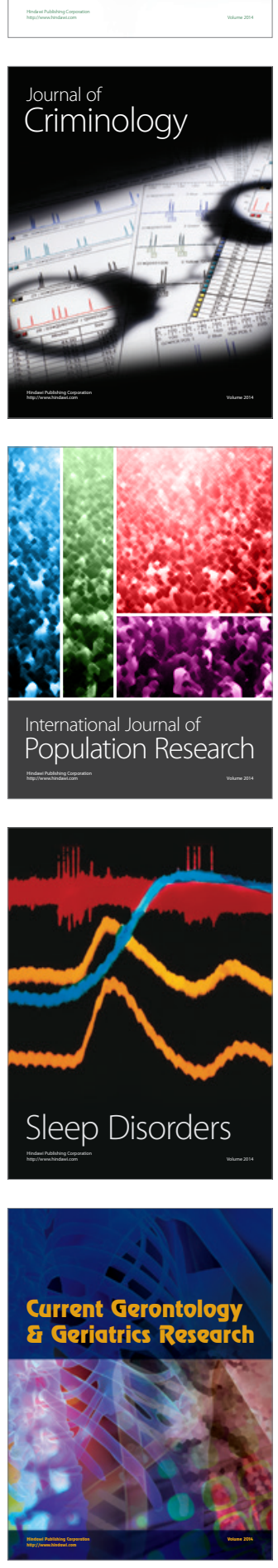
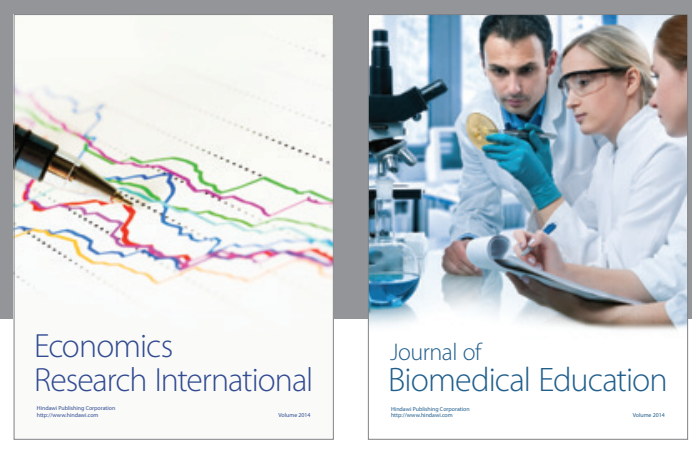

Journal of

Biomedical Education

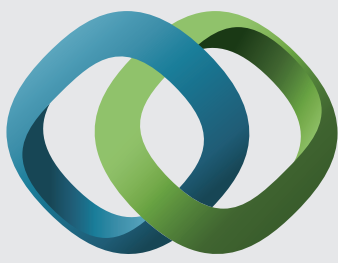

\section{Hindawi}

Submit your manuscripts at

http://www.hindawi.com
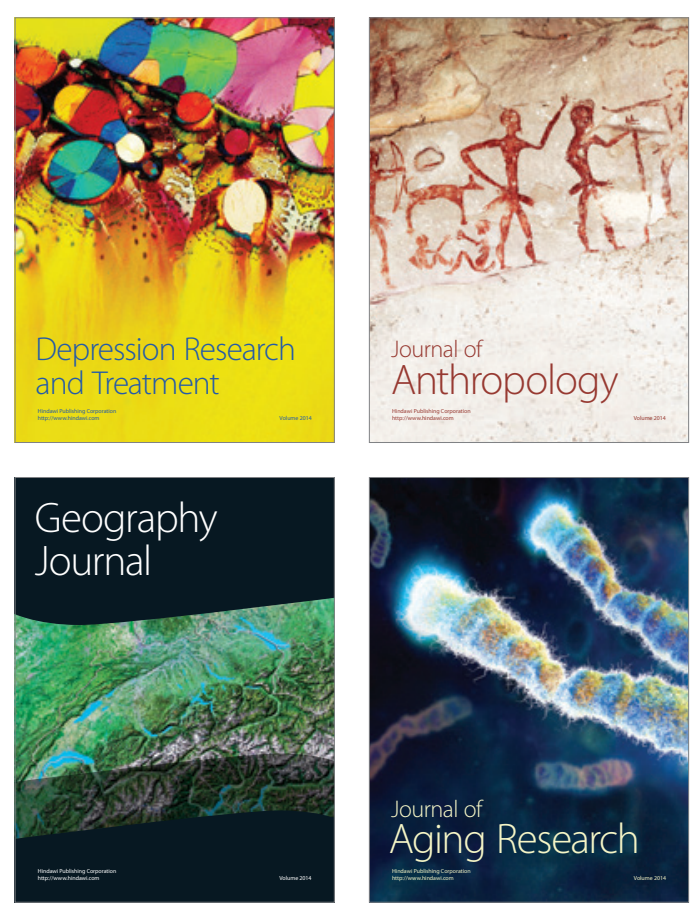

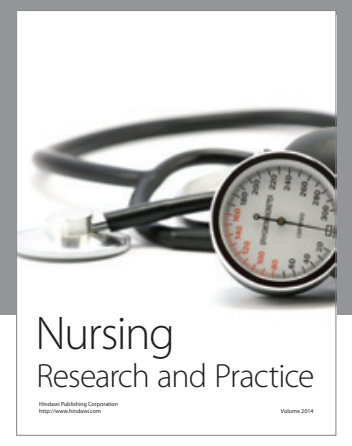

Nursing

Research and Practice

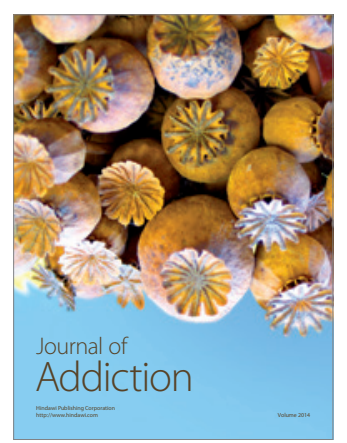

Child Development

Research

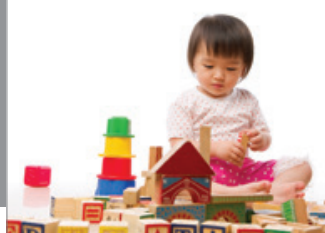

迥
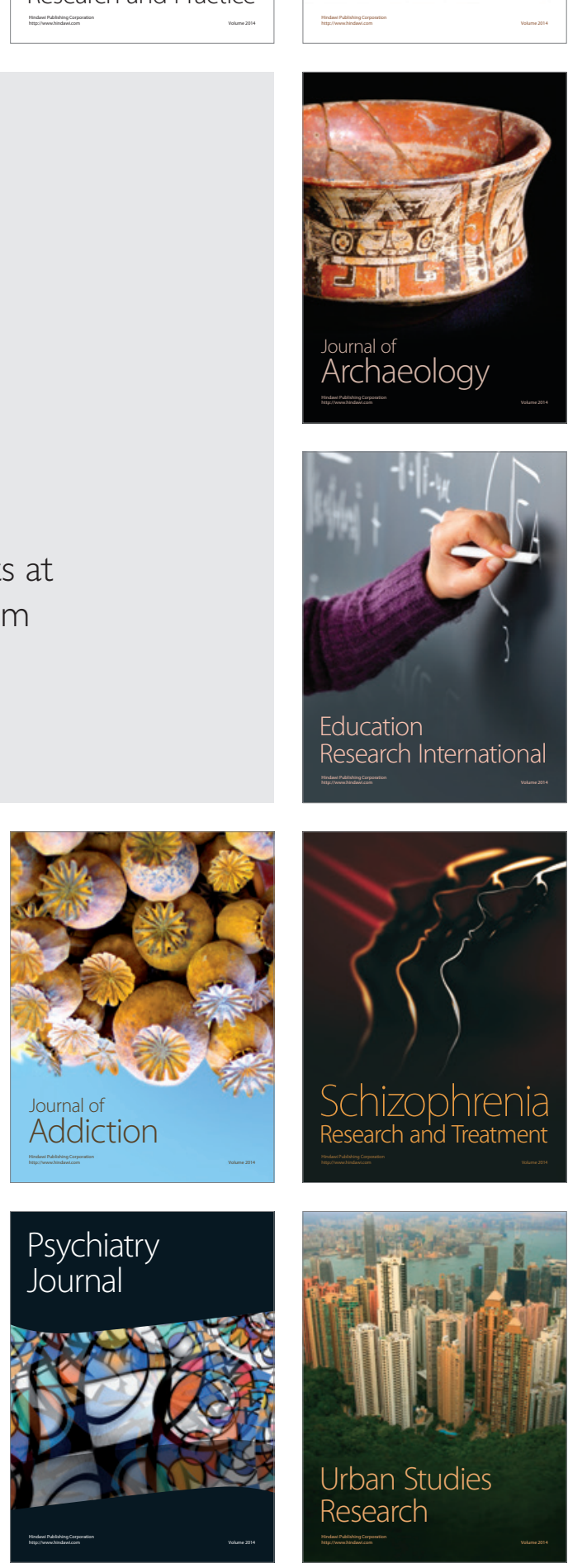